\title{
A COMPARATIVE STUDY OF PLASMA EXPANSION EVENTS IN THE POLAR WIND
}

TAMAS I. GOMBOSI

Space Physics Research Laboratory, The University of Michigan, Ann Arbor, MI 48109, U.S.A.

and

ROBERT W. SCHUNK

Center for Atmospheric and Space Sciences, Utah State University, Logan, UT 84322-4405, U.S.A.

\author{
(Received 8 December 1987)
}

\begin{abstract}
The temporal characteristics of the polar wind were studied with both a full set of hydrodynamic equations and a simplified set of collisionless equations. The hydrodynamic set contained continuity, momentum and energy equations for $\mathrm{H}^{+}, \mathrm{O}^{+}$and electrons, including collisional conductivities and variable, but isotropic, ion and electron temperatures. The collisionless model included the same species and was able to take account of constant anisotropic ion temperatures. The two polar wind models were solved with different numerical techniques and the results were compared for similar expansion scenarios. In particular, the temporal evolution of the polar wind was modelled for initial, extended density depletions of factors of 3, 10 and 100 starting at several altitudes. Also, the two models were used to study the temporal response of the polar wind to varying electron temperature conditions. From this comparative study, we found the following: (1) qualitatively, the two models predict the same temporal characteristics for the polar wind densities, flow velocities, and escape fluxes; (2) the propagation velocities of the disturbances vary markedly with the level of the depletion, with greater depletions producing greater disturbance velocities; (3) for small depletions the $\mathrm{H}^{+}$disturbance does not steepen into a shock ; (4) the rapidly moving $\mathrm{H}^{+}$disturbances do not significantly affect the $\mathrm{O}^{+}$distribution, but the slower moving $\mathrm{O}^{+}$ shocks generate secondary $\mathrm{H}^{+}$disturbances that propagate with the heavy ion shock waves; (5) for a 100 fold depletion, the rapid acceleration of the minor $\mathrm{H}^{+}$ions through the $\mathrm{O}^{+}$shock acts to enhance the $\mathrm{H}^{+}$ density at higher altitudes to such an extent that $\mathrm{H}^{+}$becomes the dominant ion over a limited altitude range ahead of the $\mathrm{O}^{+}$shock; (6) the adiabatic cooling associated with the upward propagation of the disturbances leads to a significant reduction in the $\mathrm{O}^{+}$temperature, and a less dramatic reduction in the $\mathrm{H}^{+}$temperature; and (7) the polar wind expansion characteristics are strongly modulated when the electron temperature variations are of the order of five minutes. The density distribution becomes highly structured, and regions of upward and downward ion flows follow the temporal variations of $T_{e}$. Such a situation can occur when the interplanetary magnetic field is northward and the magnetospheric convection pattern is turbulent.
\end{abstract}

\section{INTRODUCTION}

In the early 1960s, it was recognized that the geomagnetic field lines at high latitudes are not dipolar, but instead extend well beyond the orbit of the moon in the antisunward direction (Axford and Hincs, 1961). Soon after this discovery, it was suggested that thermal evaporation of the light ions $\left(\mathrm{H}^{+}\right.$and $\left.\mathrm{He}^{+}\right)$ from the topside ionosphere should lead to a continual outflow of plasma from the polar regions (Dessler and Michel, 1966; Bauer, 1966). However, it was subsequently argued that the outflow should be supersonic and it was termed the "polar wind" in analogy to the solar wind (Axford, 1968). A simple hydrodynamic model was then used that emphasized the supersonic nature of the flow, thereby elucidating its basic characteristics (Banks and Holzer, 1968, 1969; Marubashi, 1970).
The supersonic nature of the polar wind has now been clearly established by both direct and indirect methods (Hoffman and Dodson, 1980; Nagai et al., 1984; Gurgiolo and Burch, 1985; Persoon et al., 1983). Also, theoretical modelling has shown that the plasma outflow is collision-dominated at low altitudes $(\leqslant 2000 \mathrm{~km})$ and collisionless at high altitudes. The collision-dominated characteristics of the flow have been extensively studied over the years with the aid of hydrodynamic models (Banks and Holzer, 1968, 1969; Marubashi, 1970; Raitt et al., 1975, 1977; Schunk et al. 1978). When the collisions prevail, the temperature (or pressure) distribution is isotropic and the heat flow is proportional to the temperature gradient. In the collisionless regime, on the other hand, the temperature distribution is anisotropic and the mathematical formulation of the polar wind is considerably more complicated. This collisionless regime 
has been modelled with hydromagnetic (Holzer et al., 1971), kinetic (Lemaire, 1972; Lemaire and Scherer, 1970, 1973), and semi-kinetic (Barakat and Schunk, 1983, 1984) equations. Also, generalized transport equations have been used that include both collisiondominated and collisionless characteristics of the ion outflow, and hence, provide for continuity from the collision-dominated to collisionless regimes (Schunk and Watkins, 1981, 1982; Demars and Schunk, 1986, 1987; Ganguli, 1986; Ganguli and Palmadesso, 1987 ; Ganguli et al., 1987).

All of the above studies of the "classical" polar wind were for "steady state" conditions, with the emphasis on elucidating physical processes. Because of these studies, significant progress has been made in understanding the basic characteristics of the outflow for a wide range of conditions (cf. Schunk, 1986, 1987). More recently, attention has been focused on studying the temporal characteristics of the polar wind. However, because of the severe numerical difficulties associated with solving the more complicated sets of transport equations (cf. Schunk, 1977; Demars and Schunk, 1979; Barakat and Schunk, 1982), the initial time-dependent models of the classical polar wind were based on hydrodynamic equations.

Singh and Schunk (1985) studied the temporal evolution of density perturbations in the supersonic collisionless polar wind, including extended density depletions and localized density bumps and holes. For this study, the $\mathrm{H}^{+}$continuity and momentum equations were solved without allowance for collisions and assuming constant, isotropic ion and electron temperatures of $3560 \mathrm{~K}$. Also, the effects of localized ion heating on the polar wind were studied by Gombosi et al. $(1985,1986)$ and by Gombosi and Killeen (1987). Both high-altitude and low-altitude ion heating events were considered. In these studies, the full hydrodynamic continuity, momentum and energy equations for $\mathrm{H}^{+}, \mathrm{O}^{+}$and electrons were solved including collisional conductivities and assuming isotropic, but variable, ion and electron temperatures.

In this paper, we continue our study of the temporal characteristics of the polar wind. First, the collisionless model described above is extended to include both $\mathrm{H}^{+}$and $\mathrm{O}^{+}$ions and the constant isotropic ion temperature is replaced with separate equations of state for the parallel and perpendicular (to $B$ ) degrees of freedom. This latter change allows us to take account of the extremely large ion temperature anisotropies that develop at high altitudes in the polar wind (cf. Holzer et al., 1971; Lemaire and Scherer, 1973), which were shown to be stable with respect to the excitation of electrostatic waves by Barakat and
Schunk (1987). The improved collisionless model and the collision-dominated model are then used to study the temporal evolution of the polar wind in response to extended density depletions starting at different altitudes in order to determine whether or not there are qualitative differences in the temporal characteristics predicted by the collisionless and collisiondominated models. The models are also used to study the temporal response of the polar wind to varying electron temperature conditions. This latter study complements those described above involving ion heating events.

\section{THEORETICAL FORMULATION}

The full hydrodynamic and simplified collisionless models used in this study are described in the following subsections.

\subsection{Hydrodynamic model}

This numerical model simultaneously solves the time-dependent coupled continuity, momentum and energy equations for $\mathrm{O}^{+}$and $\mathrm{H}^{+}$ions along diverging open magnetic field lines (the cross-sectional area of a magnetic flux tube, $A$, varies as $A \sim B^{-1}$, where $B$ is the magnetic field strength). It is assumed that the ion and electron gases can be considered to be perfect fluids with internal degrees of freedom. The plasma is assumed to be quasineutral, and no vertical electric currents are allowed $\left[n_{\mathrm{e}}=n\left(\mathrm{O}^{+}\right)+n\left(\mathrm{H}^{+}\right)\right.$and $u_{\mathrm{e}} n_{\mathrm{e} \text {. }}$ $=u\left(\mathrm{O}^{+}\right) n\left(\mathrm{O}^{+}\right)+u\left(\mathrm{H}^{+}\right) n\left(\mathrm{H}^{+}\right)$, where $u$ represents the field-aligned bulk velocity and $n$ is the particle number density]. The governing equations of this models were described in earlier papers (Gombosi et al., 1985, 1986) but unfortunately, several misprints appeared in the first paper and therefore we summarize the equations again :

$$
\frac{\partial}{\partial t}\left(A \rho_{i}\right)+\frac{\partial}{\partial r}\left(A \rho_{i} u_{i}\right)=A S_{i}
$$

$$
\begin{aligned}
\frac{\partial}{\partial t}\left(A \rho_{i} u_{i}\right)+\frac{\partial}{\partial r} & \left(A \rho_{i} u_{i}^{2}\right)+A \frac{\partial p_{i}}{\partial r} \\
& =A \rho_{i}\left(\frac{\mathrm{e}}{m_{i}} E_{\|}-g\right)+A \frac{\delta M_{i}}{\delta t}+A u_{i} S_{i}
\end{aligned}
$$

$$
\begin{array}{r}
\partial\left(\begin{array}{l}
1 \\
\partial t
\end{array} \rho_{i} u_{i}^{2}+\frac{1}{\gamma_{i}-1} A p_{i}\right)+\frac{\partial}{\partial r} \\
\times\left(\frac{1}{2} A \rho_{i} u_{i}^{3}+\frac{\gamma_{i}}{\gamma_{i}-1} A u_{i} p_{i}\right) \\
=A \frac{\delta E_{i}}{\delta t}+A Q_{i}+\frac{\partial}{\partial r}\left(A \kappa_{i} \frac{\partial T_{i}}{\partial r}\right)
\end{array}
$$




$$
\begin{gathered}
+A \rho_{i} u_{i}\left(\frac{\mathrm{e}}{m_{i}} E_{\|}-g\right)+A u_{i} \frac{\delta M_{i}}{\delta t}+\frac{1}{2} A u_{i}^{2} S_{i} \\
\rho_{\mathrm{e}} \frac{\partial T_{\mathrm{e}}}{\partial t}=\frac{\left(\gamma_{\mathrm{e}}-1\right) m_{\mathrm{e}}}{k A} \frac{\partial}{\partial r}\left(A \kappa_{\mathrm{e}} \frac{\partial T_{\mathrm{e}}}{\partial r}\right)-\rho_{\mathrm{e}} u_{\mathrm{e}} \frac{\partial T_{\mathrm{e}}}{\partial r} \\
-T_{\mathrm{e}}\left[S_{\mathrm{e}}+\frac{A^{\prime}}{A}\left(\gamma_{\mathrm{e}}-1\right) \rho_{\mathrm{e}} u_{\mathrm{e}} \frac{\partial u_{\mathrm{e}}}{\partial r}\right] \\
+\left(\gamma_{\mathrm{e}}-1\right)\left(Q_{\mathrm{e}}+\frac{\delta E_{\mathrm{e}}}{\delta t}\right)
\end{gathered}
$$$$
E_{\|}=-\frac{1}{e n_{\mathrm{e}}} \frac{\partial}{\partial r}\left[\left(p_{\mathrm{e}}+u_{\mathrm{e}}^{2} \rho_{\mathrm{e}}\right)+\sum_{\text {ions }} \frac{m_{\mathrm{e}}}{m_{i}}\left(p_{i}+u_{i}^{2} \rho_{i}\right)\right.
$$$$
\left.-\sum_{\text {ions }} \frac{m_{\mathrm{e}}}{m_{i}}\left(u_{\mathrm{e}}-u_{i}\right) S_{i}-\left(\frac{\delta M_{\mathrm{e}}}{\delta t}-\sum_{\text {ions }} \frac{m_{\mathrm{e}}}{m_{i}} \frac{\delta M_{i}}{\delta t}\right)\right]
$$$$
-\frac{1}{\mathrm{e} n_{\mathrm{e}}} \frac{A^{\prime}}{A}\left(u_{\mathrm{e}}^{2} \rho_{\mathrm{e}}-\sum_{\text {ions }} \frac{m_{\mathrm{e}}}{m_{i}} u_{i}^{2} \rho_{i}\right)
$$

where $t$ is time, $r$ is distance along magnetic field lines, $m$ is the particle mass, $\rho=m n, p$ is the pressure, $T$ is the temperature, $e$ is the electron charge, $k$ Boltzmann's constant, $\gamma$ is the specific heat ratio, $\kappa$ is the heat conductivity, $S$ is the net mass production rate, $E_{\|}$is the polarization electric field, $\delta M / \delta t$ is the momentum exchange rate, $\delta E / \delta t$ is the energy exchange rate and $Q$ is the external heating rate. The " $i$ " subscript refers to either $\mathrm{H}^{+}$or $\mathrm{O}^{+}$ions, while the "e" subscript denotes the electron gas.

The adopted neutral atmosphere model includes $\mathrm{N}_{2}, \mathrm{O}_{2}, \mathrm{O}$ and $\mathrm{H}$ (Banks and Kockarts, 1973). The $\mathrm{O}^{+}$ions are produced by photoionization, while $\mathrm{H}^{+}$ ions are created by charge exchange only. $\mathrm{O}^{+}$is chemically removed by reactions with $\mathrm{N}_{2}$ and $\mathrm{O}_{2}$, and $\mathrm{H}^{+}$ by charge transfer with $\mathrm{O}$. The momentum and energy transfer collision terms describing ion-ion, ion-neutral and electron-ion collisions, and the heat conductivities adopted in this model were taken from Raitt et al. (1975). The thermal conductivities are strictly valid only in the collision-dominated limit.

The model flux tube connects two infinite external reservoirs. The ions in the stationary lower reservoir (located at an altitude of $200 \mathrm{~km}$ ) are assumed to be in chemical and thermal equilibrium with the neutral atmosphere, while the electron temperature in the reservoir is $1000 \mathrm{~K}$. The upper reservoir (located at an altitude of $8000 \mathrm{~km}$ ) is taken to be a stationary, low pressure medium, in effect representing a topside "vacuum cleaner". A topside downward electron heat flux of $5 \times 10^{-3} \mathrm{ergs} \mathrm{cm}^{-2} \mathrm{~s}^{-1}$ is used to simulate energy deposition from the magnetosphere. It was also assumed that there is no ion heat flow between the upper reservoir and the ionospheric flux tube. The initial condition is a steady plasma outflow along open magnetic field lines. At $t=0$ the high altitude ( $>1000$ $\mathrm{km}$ ) ion densities are artificially depleted by a factor of $f$ ( $f$ varied between 3 and 100) to generate local plasma expansion events.

The seven, coupled, time-dependent partial differential equations are solved with a combined Godunov scheme/Crank-Nicholson method, which is capable of solving parabolic partial differential equation systems with propagating shock waves and other discontinuities.

\subsection{Collisionless model}

The simple collisionless model is based on the following ion equations:

$$
\begin{gathered}
\frac{\partial}{\partial t}\left(A \rho_{i}\right)+\frac{\partial}{\partial r}\left(A \rho_{i} u_{i}\right)=0 \\
\rho_{i}\left[\frac{\partial u_{i}}{\partial t}+\frac{\partial}{\partial r}\left(\frac{u_{i}^{2}}{2}\right)\right]+\frac{\partial}{\partial r}\left(n_{i} \mathrm{k} T_{i}^{\|}\right)-n_{i} \mathrm{e}_{i} E_{\|} \\
+\rho_{i} \frac{\mathrm{G} M_{E}}{r^{2}}+n_{i} \mathrm{k}\left(T_{i}^{\|}-T_{i}^{\perp}\right) \frac{1}{A} \frac{\partial A}{\partial r}=0 \\
T_{i}^{\|} n_{i}^{\left(1-y_{i}\right)}=\text { constant } \\
T_{i}^{\perp} / B=\text { constant }
\end{gathered}
$$

where $T_{i}^{\|}$and $T_{i}^{\perp}$ are the ion temperatures parallel and perpendicular to $B$, respectively, $G$ is the gravitational constant, $M_{E}$ is the Earth's mass, $(1 / A) \partial A / \partial r=3 / r$, and $r$ is geocentric distance. As with the previous model, the subscript $i$ refers to either $\mathrm{H}^{+}$ or $\mathrm{O}^{+}$ions.

The polarization electric field is obtained from the electron momentum equation,

$$
E_{\sharp}=-\frac{1}{\mathrm{e} n_{\mathrm{e}}} \frac{\partial p_{\mathrm{e}}}{\partial r}
$$

where $p_{\mathrm{e}}=n_{\mathrm{e}} \mathrm{k} T_{\mathrm{e}}$ and where $T_{\mathrm{e}}$ is assumed to be governed by an equation of state,

$$
T_{\mathrm{e}} n_{\mathrm{e}}^{\left(1-\gamma_{\mathrm{e}}\right)}=\text { constant. }
$$

Note that equations (10) and (5) are equivalent, since the additional terms in equation (5) are negligible for polar wind applications (Raitt et al., 1975; Schunk and Watkins, 1981). As with the full hydrodynamic model, the electron density and drift velocity are obtained by assuming charge neutrality and charge conservation with no field-aligned current.

The above set of nonlinear, time-dependent partial differential equations are solved with initial conditions 
and boundary values using the well-known flux-corrected-transport (FCT) technique (Boris and Book, 1976). The equations are solved along a diverging magnetic flux tube from a lower boundary $r_{0}$ to a specified upper boundary. At the lower boundary, the ion densitics and drift velocitics are specified, while at the upper boundary an outflow condition is assumed. For perturbation studies, our initial ion density and drift velocity profiles are obtained from steady-state solutions of the above equations.

\section{PLASMA EXPANSION ChaRACTERISTICS}

In this section we show the temporal characteristics of the polar wind in response to different perturbations, including extended density depletions, timevarying electron temperature conditions, and anisotropic ion temperatures. The different situations were modelled using both the full set of hydrodynamic equations as well as simplified collisionless equations, and consequently, a comparison of the two approaches was also obtained. Since the two models are different, it is convenient to present the results in separate subsections.

Before presenting results, we note that some work has already been done with regard to extended density depletions (Singh and Schunk, 1985). This work, however, was very restricted, with the main goal being a comparison of macroscopic plasma expansion characteristics with those predicted by microscopic formulations (i.e. expansions on a Debye-length scale). Specifically, only three cases were considered by Singh and Schunk (1985). In all three cases, the extended depletions were very severe (factor of $10^{3}$ or $10^{5}$ ) and the ion and electron temperatures were constant at $3560 \mathrm{~K}$. Two of the cases were "single-ion" expansions with the extended depletions starting at high altitudes $(r \geqslant 12,000 \mathrm{~km})$, and the third case was a multi-ion expansion with the extended depletion at $r \geqslant 9188$ $\mathrm{km}$. Here, we conducted a more systematic study, considering extended depletions of factors of 3,10 and 100 , which better represent the magnitudes of the density perturbations expected to occur in the polar wind. We also considered a range of altitudes from $1000 \mathrm{~km}$, where collisions are important, to $6000 \mathrm{~km}$, where the polar wind is collisionless. In addition, we used both the full hydrodynamic formulation, which includes self-consistent temperature variations, and simplified collisionless equations, which take account of the effect of constant anisotropic ion temperatures.

\subsection{Hydrodynamic results}

The results obtained from the full hydrodynamic formulation for extended density depletions above
$1000 \mathrm{~km}$ of factors of 3,10 and 100 are shown in Figs la-d. The starting conditions correspond to typical steady state polar wind solutions. Then, at $t=0$, the plasma densities above $1000 \mathrm{~km}$ are reduced by a given factor, while keeping the topside electron heat flux at the previous level $\left(5 \times 10^{-3} \mathrm{erg} \mathrm{cm}^{-2} \mathrm{~s}^{-1}\right)$, thus initiating a plasma expansion event without significant changes in the electron temperature structure. Inspection of the density, drift velocity, temperature and Mach number profiles shown in Figs la-d reveals several interesting features. First, the propagation velocities of the disturbances vary markedly with the level of depletion, with greater depletions producing higher disturbance velocities. Also, the light ion $\left(\mathrm{H}^{+}\right)$ disturbance propagates faster than the heavy ion $\left(\mathrm{O}^{+}\right)$ disturbance. For the 3-fold depletion, the $\mathrm{H}^{+}$and $\mathrm{O}^{+}$ disturbances propagate with about 15 and $3.6 \mathrm{~km} \mathrm{~s}^{-1}$, respectively. For the 10 -fold depletion, these disturbance velocities are 23 and $4.4 \mathrm{~km} \mathrm{~s}^{-1}$, while for the 100 -fold depletion they are 30 and $5.2 \mathrm{~km} \mathrm{~s}^{-1}$. These general trends are consistent with those found by Singh and Schunk (1985) for the three severe depletions they considered.

However, the expansion characteristics shown in Figs 1a-d differ significantly from the "severe depletion" characteristics in several ways. First, for the 3fold depletion, the $\mathrm{H}^{+}$disturbance does not steepen into a shock, and for the 10-fold and 100-fold depletions the $\mathrm{H}^{+}$fronts are not that steep. However, for $\mathrm{O}^{+}$, all three depletions steepen into well-defined shocks. Also, the $\mathrm{H}^{+}$disturbances move very rapidly through the topside ionosphere without significantly modifying the heavy ion $\left(\mathrm{O}^{+}\right)$properties, while the modifications of the polarization electric field associated with the slower moving $\mathrm{O}^{+}$shocks are sufficient to generate secondary $\mathrm{H}^{+}$disturbances that propagate with the heavy ion shock waves. [Further comparisons to that one multi-ion, severe depletion of Singh and Schunk (1985) are not possible since they only followed the temporal evolution for $249 \mathrm{~s}$.]

There are other interesting new features shown in Figs 1a-d. For the 100-fold depletion, there is a rapid acceleration of the minor $\mathrm{H}^{+}$ions through the $\mathrm{O}^{+}$ shock, which leads to reduced $\mathrm{H}^{+}$densities in and below the $\mathrm{O}^{+}$shock and enhanced $\mathrm{H}^{+}$densities above it (see $t=100,200$ and $500 \mathrm{~s}$ ). The net result is that $\mathrm{H}^{+}$becomes the dominant ion at high altitudes for a brief period of time. With regard to the thermal structure, the adiabatic cooling associated with the upward propagation of the $\mathrm{O}^{+}$disturbance leads to a significant reduction in the $\mathrm{O}^{+}$temperature for all three depletion cases. For $\mathrm{H}^{+}$, on the other hand, the temperature is not cooled to the same extent as $\mathrm{O}^{+}$ owing to the higher $\mathrm{H}^{+}$conductivity, and for the $100-$ 

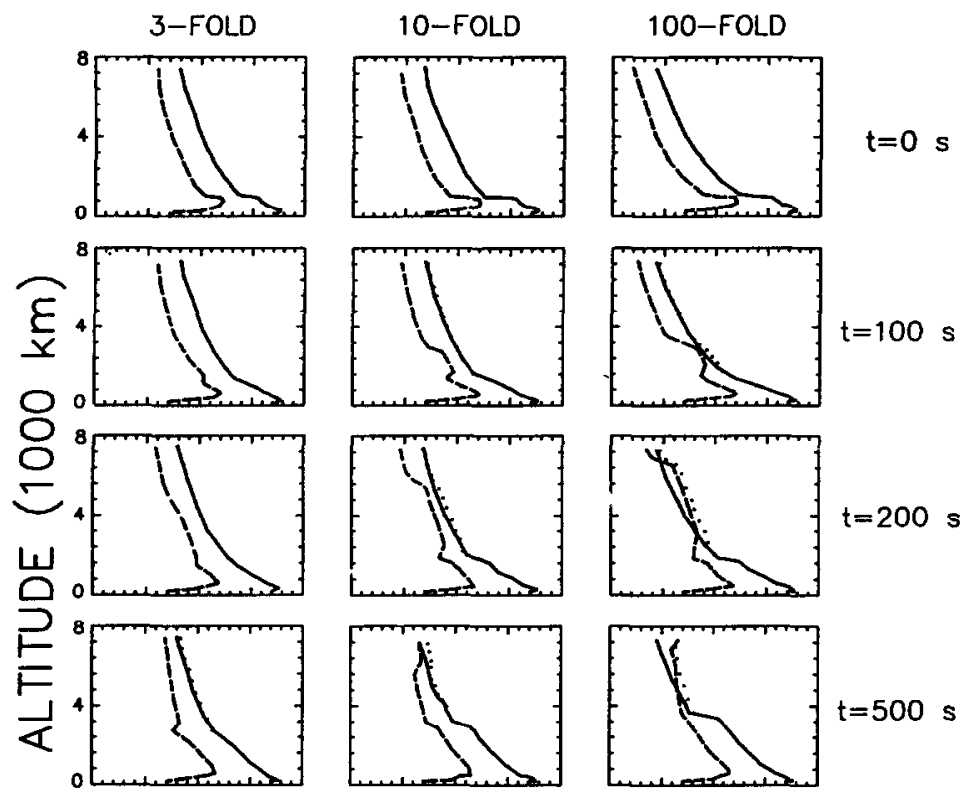

$$
t=200 s
$$
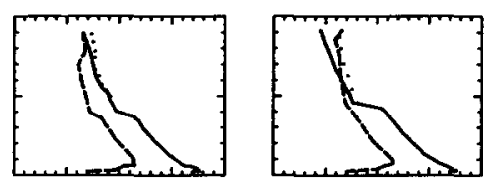

$t=500 s$
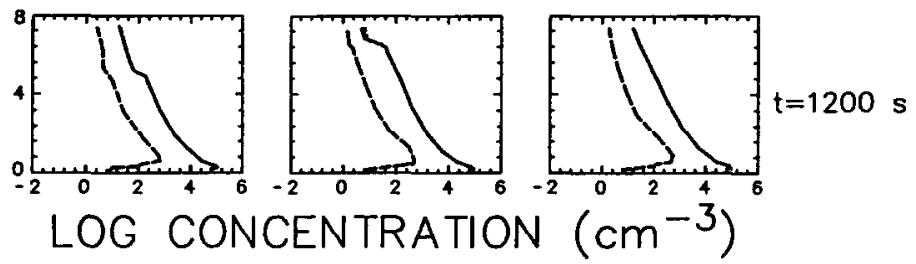

Fig. 1a. Temporal eVOlution OF THE $\mathrm{H}^{+}$(DASHEd CURVES), $\mathrm{O}^{+}$(SOlid CURVES) AND ELECTRON (DOTTED CURVES) DENSITY PROFILES IN RESPONSE TO EXTENDED DENSITY DEPLETIONS ABOVE $1000 \mathrm{~km}$ OF FACTORS OF 3 (LEFT COLUMN), 10 (MIDDLE COLUMN) AND 100 (RIGHT COLUMN).

fold density depletion there is even a transient increase of the $\mathrm{H}^{+}$temperature just after the $\mathrm{O}^{+}$shock crosses the upper boundary $(t=1200 \mathrm{~s})$.

It is anticipated that the polar wind expansion characteristics should be modulated by fluctuations in the thermal electron temperature, since it directly affects the polarization electric field. However, because of the presence of magnetospheric electric fields and energetic particle precipitation, the variation of the electron temperature can be very complex, depending on $K_{p}$ and the interplanetary magnetic field (IMF). When the IMF is southward, two-cell plasma convection occurs with antisunward flow over the polar cap and return flow equatorward of the auroral oval. In this case, convecting flux tubes of plasma would experience changing $T_{\mathrm{e}}$ conditions as they move into and out of sunlight, the auroral oval, and Joule heating events. When the IMF is northward, on the other hand, multiple convection cells or turbulent convection are possiblc, and thesc features are accompanied by sun-aligned arcs and a general level of polar cap precipitation. In this case, the convecting flux tubes of plasma will experience a wide variety of thermal electron heating events as they move around the polar region.

In order to study the possible effect of such heating events on the polar wind, we simulated a few idealized electron heating events. Figure 2 shows the temporal evolution of the plasma parameters following an initial 10-fold density depletion combined with a cutoff of the topside electron heat flux. In this case, the plasma expansion event is superimposed on a collapsing thermal structure of the topside ionosphere. Initially $(t<500 \mathrm{~s})$, the plasma motions are very close to what can be seen in Fig. 1. In about $500 \mathrm{~s}$ the electron temperature profile reaches its new equilibrium $(\sim 1000 \mathrm{~K})$ and becomes smaller than the ion temperatures. The ion temperatures vary with a much longer time scale, therefore, the ions start to feel the consequences of the decreasing temperatures only around $10 \mathrm{~min}$ after the initiation of the expansion event. At $t=500 \mathrm{~s}$, there is a downward $\mathrm{O}^{+}$flow 


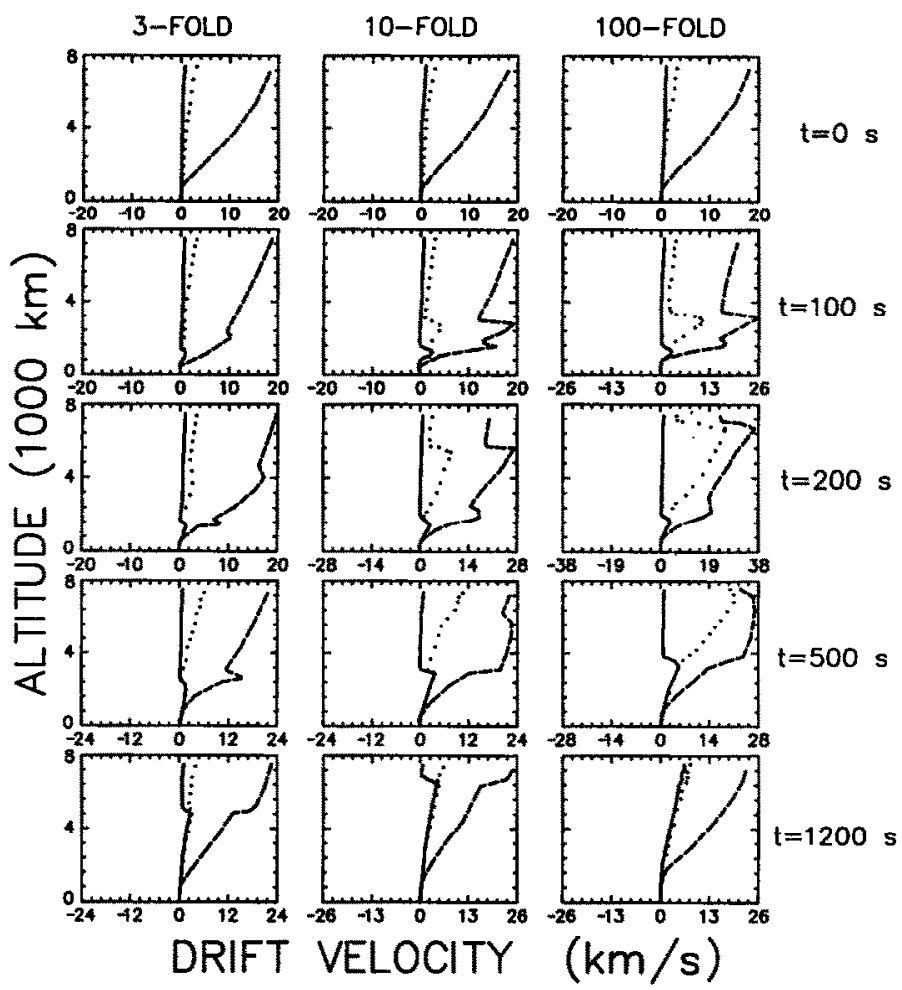

Fig. Ib. TEMPORAL EVOLUTION OF THE $\mathrm{H}^{+}, \mathrm{O}^{+}$AND ELECTRON DRIFT VELOCITY PROFILES THAT CORRESPOND TO THE DENSITY PROFILES SHOWN IN FIG. 1a.

between $\sim 2500$ and $6000 \mathrm{~km}$ which transports heavy ions to the colder lower region. At $1200 \mathrm{~s}$, an interesting situation can be observed. The downward $\mathrm{O}^{+}$ flow is stopped by the denser region and most of the flow energy is converted into heat, thus resulting in a transient heating of the low-altitude heavy ion population. At the same time the leading edge of the rapidly eroding $\mathrm{O}^{+}$upward shock is still present around 3500 $\mathrm{km}$, causing the interesting double downflow structure. About an hour after the beginning of the event, the upflowing heavy ion structure is replaced by the downflowing $\mathrm{O}^{+}$structure. At around $1800 \mathrm{~km}$, an $\mathrm{H}^{+}-\mathrm{O}^{+}$crossover can be seen which later gradually moves upward. After about $3 \mathrm{~h}$ the ionosphere reaches a new equilibrium with cold temperature profiles and much smaller ionospheric scale-heights.

To simulate the highly variable electron heating that might occur as flux tubes of plasma move in random directions into and out of precipitation zones when the IMF is northward, we periodically turned the topside electron heating on and off during an expansion event. At $t=0$, a 10 -fold extended density depletion was initiated and the topside electron heat flux was shut off. At $t=300 \mathrm{~s}$, the heat flux was turned on again for $5 \mathrm{~min}$. This topside energy pumping was periodically activated for $5 \mathrm{~min}$ at subsequent 10 -min intervals. The effect on the plasma parameters, which is shown in Fig. 3, is that even though the electron temperature responds rapidly following every cutoff of the topside energy source, the ion temperature has a much longer time constant. Therefore, regions of upward and downward ion flows follow each other periodically and the ion density profiles also become structured. Consequently, when the IMF is northward and turbulent convection occurs, the polar wind should be highly structured.

\subsection{Collisionless results}

Steady state polar wind solutions indicate that the flow becomes collisionless in the $2000-3000 \mathrm{~km}$ altitude range (cf. Holzer et al., 1971; Lemaire and Scherer, 1973; Raitt et al., 1975). In the collisionless regime, large ion temperature anisotropies develop and the collision-dominated thermal conductivities may not be appropriate (cf. Schunk and Watkins, 1982). Although the appropriate set of plasma transport equations has been derived (Demars and Schunk, 1979), it is extremely difficult to solve numerically. 

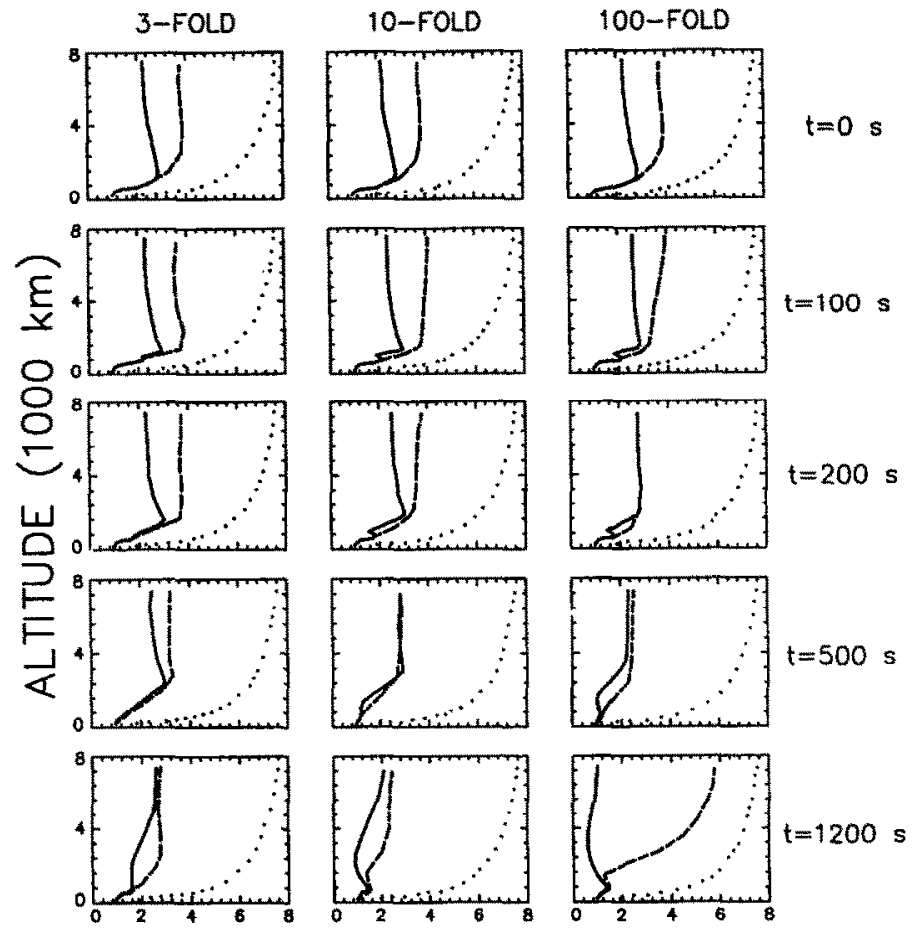

TEMPERATURE $(1000 \mathrm{~K})$

Fig. 1c. TEMPORAL EVOLUTION OF THE $\mathrm{H}^{+}, \mathrm{O}^{+}$aNd ElECTRON TEMPERATURE PROFILES THAT CORRESPOND TO THE DENSITY PROFILES SHOWN IN FIG. 1a.

Therefore, it would be useful to know whether or not the details of the thermal structure are crucial for determining the temporal evolution of the polar wind densities, drift velocities and escape fluxes. In an effort to shed light on this problem, we solved the collisionless transport equations (6)-(9), which take account of ion temperature anisotropies in a simplistic manner, for expansion conditions similar to those used with the full hydrodynamic equations.

The steady state polar wind solutions predict that the parallel ion temperatures are basically constant with altitude or decrease slowly with altitude, while the perpendicular ion temperatures decrease rapidly with altitude. These results can be simulated via equations (8) and (9) with the appropriate choice of $\gamma_{i}$. However, we found that a slight decrease in $T_{i}^{\|}$with altitude has a negligible effect on the results, so the cases we present below are based on $\gamma_{i}=1$ for both $\mathrm{H}^{+}$and $\mathrm{O}^{+}$ions.

Because the present formulation is collisionless and the thermal structure is not calculated self-consistently, an exact comparison with the hydrodynamic results cannot be made. Nevertheless, qualitative comparisons can be made by using the densities and tem- peratures obtained from the hydrodynamic formulation at $1000 \mathrm{~km}$ as boundary conditions for the collisionless solutions. From Figs $1 \mathrm{a}-\mathrm{d}$, the initial $(t=0)$ steady state polar wind parameters at $1000 \mathrm{~km}$ are $n_{0}\left(\mathrm{O}^{+}\right)=1.5 \times 10^{4} \mathrm{~cm}^{-3}, n_{0}\left(\mathrm{H}^{+}\right)=3 \times 10^{2} \mathrm{~cm}^{-3}$, $T_{0}\left(\mathrm{O}^{+}\right)=2500 \mathrm{~K}, T_{0}\left(\mathrm{H}^{+}\right)=4000 \mathrm{~K}$, and $T_{\mathrm{e} 0}=7000$ $\mathrm{K}$. Note that $T_{\mathrm{v}}$ shows very little variation with time for all three depletion cases (see Fig. 1c) and is roughly constant at $7000 \mathrm{~K}$ above $1000 \mathrm{~km}$. Therefore, we chose $\gamma_{e}=1$ in equation (11) for the collisionless simulations. The ion temperatures are obtained from equations (8) and (9) using the adopted boundary values at $1000 \mathrm{~km}$. Altitude profiles of the resulting parallel and perpendicular ion temperatures are shown in Fig. 4. Note that the total ion temperature is obtained from the expression $T_{i}=\left(T_{i}+2 T_{i}^{i}\right) / 3$, and therefore, it decreases with altitude for both $\mathrm{H}^{+}$and $\mathrm{O}^{+}$ions.

Using the adopted boundary values and the thermal structure described above, the simplified collisionless equations were first solved to obtain stcady statc polar wind profiles. Then, at $t=0$, extended density depletions of factors of 3,10 and 100 were imposed above a certain altitude while keeping the velocity profiles unchanged, and the subsequent temporal 


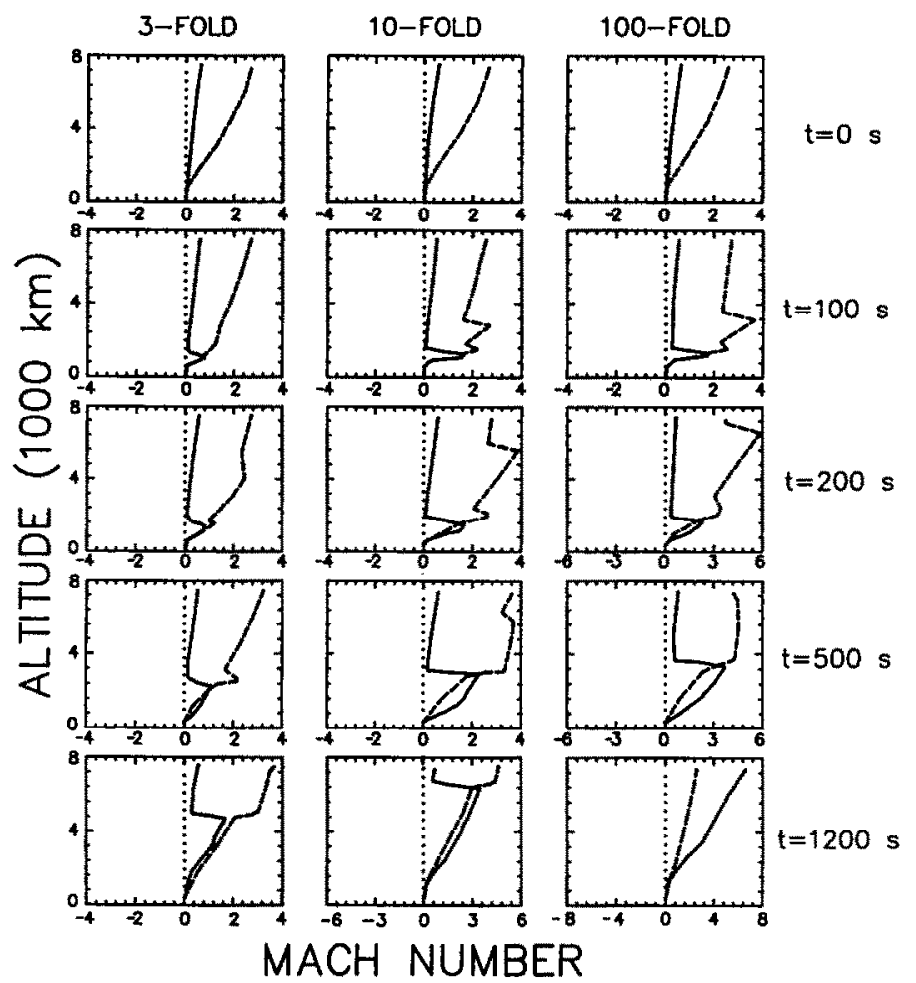

Fig. 1d. Temporal evolution of the $\mathrm{H}^{+}, \mathrm{O}^{+}$aNd electron Mach number Profiles that CoRrespond TO THE DENSITY PROFILES SHOWN IN FIG. Ia.

evolutions of the perturbations were followed until they crossed our upper boundary. However, depletions were imposed only above $2000 \mathrm{~km}$, which is the region where the collisionless equations are expected to hold. In contrast, the extended depletions simulated with the full set of hydrodynamic equations started at $1000 \mathrm{~km}$.

Figure 5 shows the temporal evolution of the $\mathrm{H}^{+}$ and $\mathrm{O}^{+}$density profiles for initial extended density depletions above $2000 \mathrm{~km}$ of factors of 3,10 and 100 . A comparison of these results with those obtained using the full set of hydrodynamic equations (Fig. 1a) indicates that they are qualitatively very similar. The similarities include the following features: (1) the propagation velocity of the disturbance is greater for larger depletions and for lighter ions; (2) for a 3-fold depletion the $\mathrm{H}^{+}$disturbance does not steepen into a shock, whereas well-defined shocks form for all three $\mathrm{O}^{+}$depletions before they cross our upper boundary; (3) the $\mathrm{H}^{+}$disturbances move rapidly through the topside ionosphere without appreciably affecting the heavy $\mathrm{O}^{+}$ion, while the $\mathrm{O}^{+}$shocks generate secondary $\mathrm{H}^{+}$disturbances; and (4) for the 100-fold depletion, there is a rapid acceleration of the minor $\mathrm{H}^{+}$ions through the $\mathrm{O}^{\prime}$ shock, which results in $\mathrm{H}^{+}$becoming the dominant ion at high altitudes for a brief period of time (see $t=100 \mathrm{~s}$ frame). The only apparent difference between the full hydrodynamic model results and the collisionless model results is that the disturbances propagate at a slightly faster speed for the collisionless model. This difference is related primarily to the different thermal structures in the models and is to be expected. Also, collisions tend to slow the disturbance velocities at low altitudes.

We also considered extended density depletions starting at 4000 and $8000 \mathrm{~km}$, but no new expansion characteristics were obtained and, hence, the results will not be presented. In addition, we conducted several expansion simulations with varying electron temperatures to complement those presented earlier for the hydrodynamic model. We considered plasma expansions with a $T_{\mathrm{e}}$ that decreased from 7000 to 1000 $\mathrm{K}$ during time periods of both 0.5 and 1 hour and with both linear and parabolic time variations. These collapsing $T_{\mathrm{e}}$ structures had a negligibly small effect on the plasma "expansion characteristics". The largest effect was shown earlier with the hydrodynamic model when the electron heating at the upper boundary was 
10 - FOLD INITAL DEPLETON: NO TOPSIDE ELECTRON HEATING

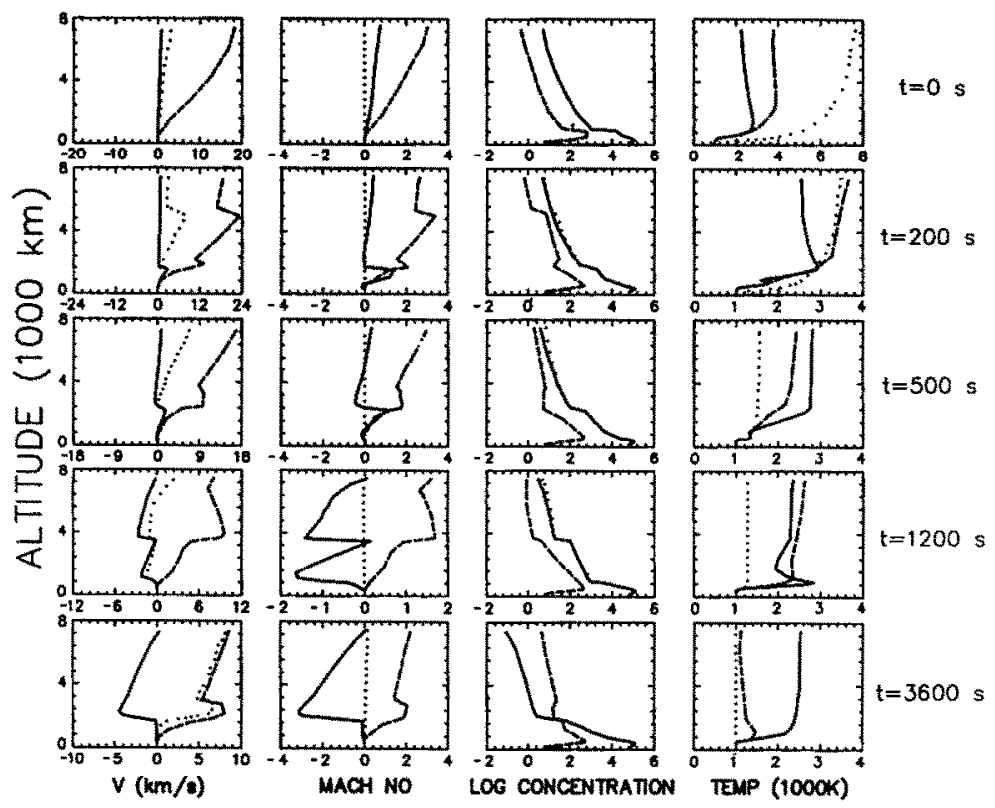

Fig. 2. TEMPoral eVolution of THE $\mathrm{H}^{+}$(DASHEd CURVES), $\mathrm{O}^{+}$(SOLID CURVES) AND Electron (DOTTED CURVES) DENSITY, DRIFT VELOCITY, MACH NUMBER AND TEMPERATURE PROFILES FOR THE CASE OF A 10-FOLD INITIAL DENSITY DEPLETION COMBINED WITH A CUIOFF OF THE TOPSIDE ELECTRON HEAT FLLX.

Note the change in the velocity, Mach number, and temperature scales as time progresses.

10 - FOLD INITAL DEPLETON MTH OSCILLATING TOPSIDE HEATING

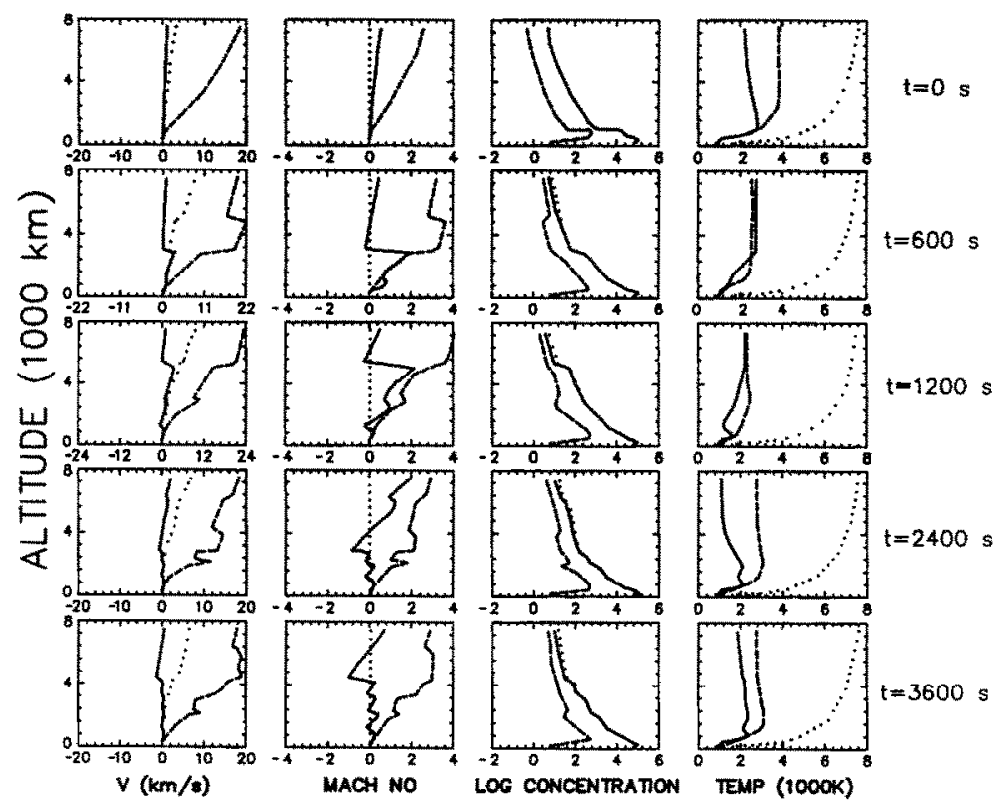

Fig. 3. Temporal evolution of THE $\mathrm{H}^{+}$(Dashed curves), $\mathrm{O}^{+}$(SOlid Curves) and electron (Dotted CURVES) DENSITY, DRIFT VELOCITY, MACH NUMBER AND TEMPERATURE PROFILES FOR THE CASE OF A 10-FOLD INITIAL DENSITY DEPLETION COMBINED WITH AN OSCILLATING TOPSIDE ELECTRON HEAT FLUX. Note the change in the velocity scale as time progresses. 


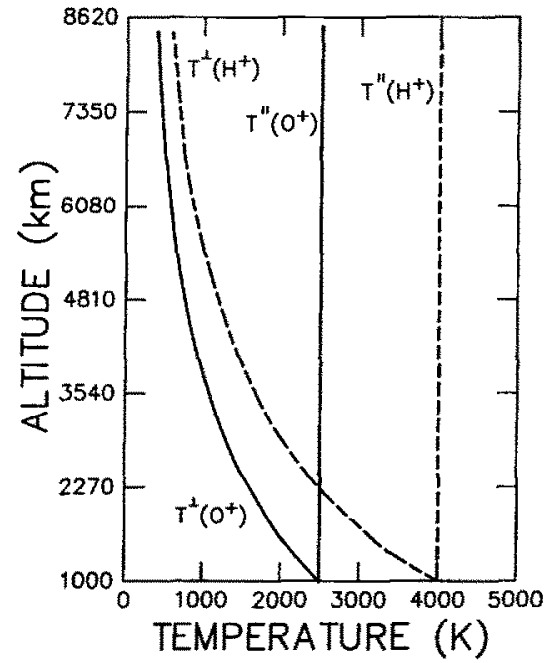

FIG, 4. ION TEMPERATURES PARALLEL AND PERPENDICULAR TO THE GEOMAGNETIC FIELD.

set to zero at $t=0$ and the electron temperature decreased at the maximum rate.

\section{SUMMARY}

The temporal characteristics of the polar wind were studied with both a full set of hydrodynamic equations and a simplified set of collisionless equations that took account of ion temperature anisotropies. The hydrodynamic set contained continuity, momentum and energy equations for $\mathrm{H}^{+}, \mathrm{O}^{+}$and electrons, including collisional conductivities and variable, but isotropic, ion and electron temperatures. The collisionless model included the same species and was able to take into account the extremely large ion temperature anisotropies that develop at high altitudes, but the temporal characteristics of the anisotropies were not modelled. The two different polar wind models were solved with different numerical techniques and the results were compared for similar expansion scenarios. Specifically, the temporal evolution of the polar wind was modelled for initial, extended density depletions of factors of 3,10 and 100 starting at several altitudes in order to determine whether or not there are qualitative differences in the polar wind characteristics predicted by the two models. The models were also used to study the temporal response of the polar wind to varying electron temperature conditions.

From the comparative study, we found the following:

(1) Qualitatively, the two models predict the same

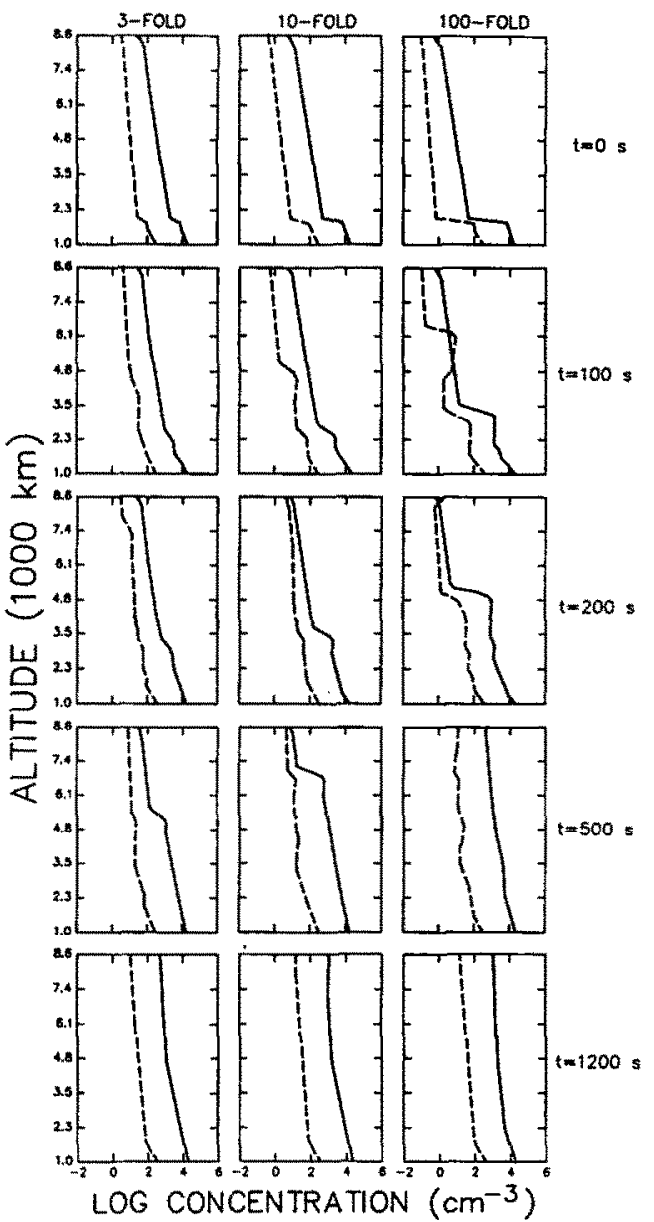

Fig. 5. Temporal evolution of THE $\mathrm{H}^{+}$(DASHed Curves) AND $\mathrm{O}^{+}$(SOLID CURVES) DENSITY PROFILES IN RESPONSE TO EXTENDED DENSITY DEPLETIONS ABOVE $2000 \mathrm{~km}$ OF FACTORS OF 3 (LEFT COLUMN), 10 (MIDDLE COLUMN) AND 100 (RIGHT COLUMN).

These profiles were calculated with the simplified collisionless transport equations.

temporal characteristics for the polar wind densities, flow velocities, and escape fluxes.

(2) The propagation velocities of the disturbances vary markedly with the level of the depletion, with greater depletions producing greater disturbance velocities.

(3) For the 3-fold depletion, the $\mathrm{H}^{+}$disturbance does not steepen into a shock. For $\mathrm{O}^{+}$, on the other hand, all three depletions steepen into well-defined shocks.

(4) The $\mathrm{H}^{+}$disturbances move rapidly through the topside ionosphere without significantly modifying the heavy $\mathrm{O}^{+}$ion distribution, while the modifications of the polarization electric field associated with the 
slower moving $\mathrm{O}^{+}$shocks are sufficient to generate secondary $\mathrm{H}^{+}$disturbances that propagate with the heavy ion shock waves.

(5) For the 100 -fold depletion, there is a rapid acceleration of the minor $\mathrm{H}^{+}$ions through the $\mathrm{O}^{+}$ shock, which acts to enhance $\mathrm{H}^{+}$densities at higher altitudes to such an extent that $\mathbf{H}^{+}$becomes the dominant ion over a limited altitude range ahead of the $\mathrm{O}^{+}$ shock.

(6) The adiabatic cooling associated with the upward propagation of the $\mathrm{O}^{+}$disturbance leads to a significant reduction in the $\mathrm{O}^{+}$temperature for all three depletion cases. For $\mathrm{H}^{+}$, however, the cooling is less dramatic, and for the 100-fold density depletion there is even a transient increase in $T\left(\mathrm{H}^{+}\right)$just after the $\mathrm{O}^{+}$shock crosses the upper boundary.

(7) Qualitatively, extended density depletions starting at altitudes of $1000,2000,4000$ and $8000 \mathrm{~km}$ have similar temporal characteristics.

(8) Electron temperature variations that occur on relatively long time scales ( $\geqslant 1 / 2$ hour) do not appreciably affect the polar wind expansion characteristics. However, the expansion characteristics are strongly modulated when the time scale for $T_{\mathrm{e}}$ variations is of the order of minutes. In this case, the polar wind density distribution can become highly structured, and regions of upward and downward ion flows follow the temporal variations of $T_{\mathrm{e}}$. Consequently, when the IMF is northward and turbulent magnetospheric convection occurs, the polar wind should be highly structured.

Acknowledegments - This research was supported by NASA grant NAG5-472 and NSF grant ATM-8508753 to the University of Michigan and NASA grant NAGW-77 to Utah State University. Acknowledgement is also made to the National Center for Atmospheric Research, which is sponsored by the National Science Foundation, for the computing time used in some of this research.

\section{REFERENCES}

Axford, W. I. (1968) The polar wind and the terrestrial helium budget. $J$. geophys. Res. 73, 6855 .

Axford, W. I. and Hines, C. O. (1961) A unifying theory of high-latitude geophysical phenomena and geomagnetic storms. Can. J. Phys. 39, 1433.

Banks, P. M. and Holzer, T. E. (1968) The polar wind. $J$. geophys. Res. 73, 6846.

Banks, P. M. and Holzer, T. E. (1969) Features of plasma transport in the upper atmosphere. J. geophys. Res. 74, 6304.

Banks, P. M. and Kockarts, G. (1973) Aeronomy. Academic Press, New York.

Barakat, A. R. and Schunk, R. W. (1982) Transport equations for multicomponent anisotropic space plasma: a review. Plasma Phys. 24, 389.

Barakat, A. R, and Schunk, R. W. (1983) $\mathrm{O}^{+}$ions in the polar wind. J.geophys. Res. 88, 7887.

Barakat, A. R. and Schunk, R. W. (1984) Effect of hot electrons on the polar wind. J. geophys. Res. 88,9771.

Barakat, A. R. and Schunk, R. W. (1987) Stability of the polar wind. J. geophys. Res. 92, 3409.

Bauer, S. J. (1966) The structure of the topside ionosphere, in Electron Density Profiles in Ionosphere and Exosphere (Edited by Frihagen, J.), p. 387. North-Holland, Amsterdam.

Boris, J. P. and Book, D. L. (1976) Solution of continuity equations by the method of flux-corrected transport, in Methods in Computational Physics (Edited by Alder, B., Fernbach, S. and Rotenberg, M.), Vol. 16, p. 85. Academic Press. New York.

Demars, H. G. and Schunk, R. W. (1979) Transport equations for multi-species plasmas based on individual biMaxwellian distributions. J. Phys. D. 12, 1051.

Demars, H. G. and Schunk, R. W. (1986) Solutions to biMaxwellian transport equations for SAR-are conditions. Planet. Space Sci. 34, 1335.

Demars, H. G. and Schunk, R. W. (1987) Comparison of solutions to bi-Maxwellian and Maxwellian transport equations for subsonic flows. J. geophys. Res. 92, 5969.

Dessler, A. J. and Michel, F. C. (1966) Plasma in the geomagnetic tail. J.geophys. Res. 71, 1421 .

Ganguli, S. B. (1986) Plasma transport in the auroral return current region. Ph.D. Thesis, Boston College.

Ganguli, S. B. and Palmadesso, P. J. (1987) Plasma transport in the auroral return current region. $J$, geophys. Res. 92, 8673.

Ganguli, S. B., Mitchell, H. G., Jr. and Palmadesso, P. J. (1987) Behavior of ionized plasma in the high latitude topside ionosphere: the polar wind. Planet. Space Sci. 35 , 703.

Gombosi, T. I., Cravens, T. E. and Nagy, A. F. (1985) A time-dependent theoretical model of the polar wind: preliminary results. Geophys. Res. Lett. $12,167$.

Gombosi, T. I., Cravens, T. E., Nagy, A. F. and Waite, J. H., Jr. (1986) Time-dependent numerical simulation of hot ion outflow from the polar ionosphere, in Ion Acceleration in the lonosphere and Magnetosphere (Edited by Chang, T.), p. 366. Geophysical Monograph 38, AGU, Washington, DC.

Gombosi, T. I. and Killeen, T. L. (1987) Effects of thermospheric motions on the polar wind : a time-dependent numerical study. $J$ geophys. Res. (submitted).

Gurgiolo, C. and Burch, J. L. (1985) Composition of the polar wind-not just $\mathrm{H}^{+}$and $\mathrm{He}^{+}$. Geophys. Res. Lett. 12, 69.

Hoffman, J. H. and Dodson, W. H. (1980) Light ion concentrations and fluxes in the polar regions during magnetically quiet times. $J$. geophys. Res. 85, 626 .

Holzer, T. E., Fedder, J. A. and Banks, P. M. (1971) A comparison of kinetic and hydrodynamic models of an expanding ion-exosphere, $J$.geophys, Res. 76, 2453.

Lemaire, J. (1972) $\mathrm{O}^{+} 、 \mathrm{H}^{+}$and $\mathrm{He}^{+}$ion distributions in a new polar wind model, J. atmos. terr. Phys. 34, 1647.

Lemaire, J. and Scherer, M. (1970) Model of the polar ionexosphere. Planet. Space Sci. 18, 103.

Lemaire, J. and Scherer, M. (1973) Kinetic models of the solar and polar winds. Rev. Geophys. Space Phys. 11, 427.

Marubashi, K. (1970) Escape of the polar-ionospheric plasma into the magnetospheric tail. Rep. Ionosph. Space Res. Japan. 24, 322.

Nagai, T., Waite, J. H., Green, J. L., Chappell, C. R., Olsen, R. C. and Comfort, R. H. (1984) First measurements of 
supersonic polar wind in the polar magnetosphere. Geophys. Res. Lett. 11, 669.

Persoon, A. M., Gurnett, D. A. and Shawhan, S. D. (1983) Polar cap electron densities from $D E 1$ plasma wave observations. J. geophys. Res. 88, 10123.

Raitt, W. I., Schunk, R. W. and Banks, P. M. (1975) A comparison of the temperature and density structure in the high and low speed thermal proton flows. Planet. Space Sci. 23, 1103.

Raitt, W. J., Schunk, R. W. and Banks, P. M. (1977) The influence of convection electric fields on thermal proton outflow from the ionosphere. Planet. Space Sci. 25, 291.

Schunk, R. W. (1977) Mathematical structure of transport equations for multispecics flows. Rev. Geophys. 15, 429.

Schunk, R. W. (1986) An updated theory of the polar wind.
Adv. Space Res. 6, 79.

Schunk, R. W. (1987) The polar wind. Proc. of the Huntsville Workshop on Plasma Models, in press.

Schunk, R. W., Raitt, W. J. and Nagy, A. F. (1978) Effect of diffusion-thermal processes on the high-latitude topside ionosphere. Planet. Space Sci. 26, 189.

Schunk, R. W. and Watkins, D. S. (1981) Electron temperature anisotropy in the polar wind. J. geophys. Res. 86, 91.

Schunk, R. W. and Watkins, D. S. (1982) Proton temperature anisotropy in the polar wind. J.geophys. Kes. 87, 171.

Singh, N. and Schunk, R. W. (1985) Temporal evolution of density perturbations in the polar wind. $J$. geophys. Res. 90,6487 . 